Preprint: Shan Zhong \& Joshua M. Pearce. Tightening the loop on the circular economy: Coupled distributed recycling and manufacturing with recyclebot and RepRap 3-D printing,Resources, Conservation and Recycling 128, (2018), pp. 48-58. doi: 10.1016/j.resconrec.2017.09.023

\title{
Tightening the Loop on the Circular Economy: Coupled Distributed Recycling and Manufacturing with Recyclebot and RepRap 3-D Printing
}

\author{
Shan Zhong and Joshua M. Pearce ${ }^{\mathrm{a}, \mathrm{b}, \mathrm{c} *}$ \\ ${ }^{a}$ Department of Materials Science \& Engineering, Michigan Technological University, \\ Houghton, US; \\ ${ }^{b}$ Department of Electronics and Nanoengineering, School of Electrical Engineering, \\ Aalto University, Espoo, Finland \\ ${ }^{c}$ Department of Electrical \& Computer Engineering, Michigan Technological \\ University, Houghton, US \\ Corresponding author*: pearce@mtu.edu
}

\begin{abstract}
A promising method of enhancing the circular economy is distributed plastic recycling. In this study plastic waste is upcycled into 3-D printing filament with a recyclebot, which is an open source waste plastic extruder. The recyclebot is combined with an open source self-replicating rapid prototyper (RepRap) 3-D printer, to enable post-consumer ABS plastic filament from computer waste to be further upcycled into valuable consumer products predesigned in the digital commons. The total electrical energy consumption for the combined process is monitored and an economic evaluation is completed. The coupled distributed recycling and manufacturing method for complex products reduces embodied energy by half, while reducing the cost of consumer products to pennies. This economic benefit provides an incentive for consumers to both home recycle and home manufacture, which tightens the loop on the circular economy by eliminating waste associated from transportation and retail. It is clear from the results that waste plastic can be significantly upcycled at the individual level using this commons-based approach. This tightening of the loop of the circular economy benefits the environment and sustainability as well as the economic stability of consumers/prosumers.
\end{abstract}

Keywords: circular economy; distributed recycling; distributed manufacturing; energy conservation; polymer recycling; sustainable development

\section{Introduction}

Over the last 50 years plastics have been used increasingly in a large range of products due to their versatility, low cost and durability (Gu and Togay, 2016; Passamonti et al., 2012). The global plastic production was 322 million tons in 2015, is growing 3.86\% per annum, and is expected to increase to 850 million tons per year by 2050 (Shen et al., 2009; Plastics Europe, 2016). This aggressive plastic production growth aggravates the pressure for waste plastic disposal and generates many well-established environmental issues. Landfill, incineration and recycling are the three main methods to treat postconsumer plastics according to the principle of waste hierarchy in increasing order of environmental responsibility (Gertsakis and Lewis, 2003; Webb et al., 2012). Incineration of plastic has the capability for energy recovery in the form of heat (Sinha et al., 2010), but large quantities of harmful compounds and greenhouse gases are emitted into the atmosphere during incineration (Zhang et al., 2004; Astrup et al., 2009). Plastics usually need more than 20 years to degrade in landfill conditions (Tansel and Banu, 2011) and plastic debris in landfill is also a source of secondary environmental pollutants (Zhang et al., 2004).

Incineration and landfill methods generate severe environmental issues, and this linear model of resource consumption that follows a "take-make-dispose" pattern has increasingly notable economic limits. High demand for resources leads to higher 
Preprint: Shan Zhong \& Joshua M. Pearce. Tightening the loop on the circular economy: Coupled distributed recycling and manufacturing with recyclebot and RepRap 3-D printing,Resources, Conservation and Recycling 128, (2018), pp. 48-58. doi: 10.1016/j.resconrec.2017.09.023

resource prices and supply disruptions, which exposes companies that follow the linear system to risks during heightened competition (MacArthur, 2013). To reduce risk, the concept of circular economy was first proposed by a Chinese scholar in 1998 with the aim to mitigate the contradiction between rapid economic growth and the shortage of raw materials and energy (Zhu, 1998). This fundamentally new model of circular economy is required to separate economic growth from resource consumption growth (Preston, 2012). A circular economy uses material symbiosis between different companies and production processes (Jacobsen 2006). The core of the circular economy is the circular flow of materials and the use of resources and energy through multiple phases (Yuan, 2006). The circular economy is beneficial to society and economy as a whole by reducing the use of the natural environment as a sink for waste and reducing the use of virgin materials for economic activities (Andersen, 2007).

Recycling, therefore, is the established best solution to treat post-consumer plastics following the goals of a circular economy (Bicket et al., 2014). However, traditional recycling can have a significant environmental impact as it demands the collection and transportation of relatively low-density waste plastics to collection centers and reclamation facilities for separation and reconstruction (Barton ta al., 1996). In centralized recycling systems the transportation usually consumes large quantities of energy with the concomitant emissions and environmental detriment (Craighill et al., 1996) and needs considerable labor to classify those post-consumer plastics (Themelis et al., 2011). In developing regions this labor is provided by waste pickers, which collect post-consumer plastic in landfills far below poverty-level wages (Hayami, et al., 2006; Wilson et al., 2006; Schenck and Blaauw, 2011; Feeley, et al., 2014).

Compared to the traditional recycling, distributed recycling (where consumers directly recycle their own waste) has the potential to reduce energy consumption because it can save the energy for transportation needed in conventional recycling (Arena, et al., 2003; Ross and Evans, 2003). A new promising method of such distributed plastic recycling is to upcycle plastic waste into 3-D printing filament with a recyclebot, which is an open source waste plastic extruder (Baechler et al., 2014). Waste plastic shards, powder or pellets are fed into the recyclebot through a hopper, and transported to the heating pipe by an auger (replacing the custom machined screw in traditional extrusion systems), which is driven by a motor. The plastic is compressed and melted in this heating pipe and can be extruded through the nozzle to form filament for fused filament fabrication (FFF)-based 3-D printing. In general, plastic recycled for 3-D printing filament is of the same type, and the process is simplified if recycling codes are granular enough to identify different kinds of plastics (Hunt et al., 2015). After classifying the plastic, it is cleaned and shredded into small pieces to improve the filament's quality by maintaining the consistency of the feed rate. The recyclebot makes filament from post-consumer plastics instead of raw materials, which can decrease by a factor of ten the embodied energy of the filament from the mining, processing of natural resources and synthesizing compared to traditional manufacturing method (Kreiger et al., 2013;2014). In addition, the recyclebot provides the potential to recycle plastics at any location so that consumers in their own homes can save money by offsetting purchased filament as well as reducing embodied energy for transportation (Kreiger et al., 2013;2014). In addition, professional waste pickers can sell filament for a substantial high value per $\mathrm{kg}$ than they earn for only sorted plastic to increase their personal income (Feeley, et al., 2014).

If the recyclebot is combined with an open source self-replicating rapid prototyper (RepRap) 3-D printer (Sells et al., 2010; Jones et al., 2011), then the postconsumer plastics can be turned into useful and more valuable products (Wittbrodt, et 
Preprint: Shan Zhong \& Joshua M. Pearce. Tightening the loop on the circular economy: Coupled distributed recycling and manufacturing with recyclebot and RepRap 3-D printing,Resources, Conservation and Recycling 128, (2018), pp. 48-58. doi: 10.1016/j.resconrec.2017.09.023

al., 2013; Redlich and Moritz, 2016). Compared to the traditional plastic manufacturing methods, like plastic injection molding, additive manufacturing with a 3-D printer has two advantages. First, a 3-D printer allows for accurate fabrication and scale models as it can directly produce complex parts by building a component in layers from 3-D digital designs with essentially no material waste (Crane et al., 2011; Gebhardt et al., 2010). Secondly, the 3-D printer can control the fill density of a product. By reducing the fill density of parts to the minimum necessary for mechanical functionality (Baich, et al., 2015), 3-D print-based manufacture can save materials, reduce energy consumption and decrease greenhouse gas emissions all which contribute to sustainability (Kreiger and Perace, 2013a; b; Ford and Despeisse, 2016). In addition, as 3-D printing can be accomplished locally (even in the homes of consumers) the transportation related energy can also be reduced (Birtchnell, et al., 2013). There is thus considerable research that has shown distributed manufacturing with 3-D printing can benefit the circular economy (Charter and Keiller, 2014; Mohr and Khan, 2015; van Wijk and van Wijk, 2015; Stahel, 2016; Despeisse, et al., 2017). The open source nature of the RepRap 3-D printer has resulted in rapid technical evolution and reductions in the cost; currently a basic polymer printing RepRap 3-D printer can be constructed for less than $\$ 500$ in parts (Anzalone, et al., 2015). Reducing the cost of 3-D printers has greatly expanded its popularity and enabled wide applicability for distributed manufacturing throughout the world for a wide range of products (Pearce et al., 2010; Mota, 2011; Richardson and Haylock, 2012; Gwamuri et al., 2015; Kietzmann et al., 2015; Pearce, 2015; Wittbrodt et al., 2015; Wittbrodt and Pearce, 2015; Petersen and Pearce, 2017a).

In order to analyze the impact of combining these two trends, this paper for the first time combines the distributed recycling method using a vertical recyclebot to make filament with distributed manufacturing using a delta RepRap to print useful products from post-consumer waste. Specifically, this study analyzes the recycling of acrylonitrile butadiene styrene (ABS) from computer waste (approximately 20 weight percent of end of life electronics (MOEA, 2001)), for the first time in such systems, into useful and valuable products. The total electrical energy consumption for the combined process is monitored and an economic evaluation is completed. These results are compared to the combination of traditional recycling and traditional manufacturing, and discussed in the context of improving the circular economy, energy conservation, greenhouse gas emission mitigation and economic benefit.

\section{Devices and methods}

\subsection{Material and energy measurements}

This project presents a distributed method to completely recycle thermoplastic into valuable consumer goods at the consumers' residence. Post-consumer ABS, ($\left.\mathrm{C}_{8} \mathrm{H}_{8} \cdot \mathrm{C}_{4} \mathrm{H}_{6} \cdot \mathrm{C}_{3} \mathrm{H}_{3} \mathrm{~N}-\right)_{\text {-n }}$, which is a versatile plastic used for a variety of durable goods, was chosen to test this method. ABS is good choice of plastic for recycling into filament because its glass transition temperature is not changed and the decomposition temperature increases slightly by $3{ }^{\circ} \mathrm{C}$ after recycling due to the decreasing of volatile monomers (Kim and Kang, 1995). The increased decomposition temperature provides a broader temperature range during the recycling process and after recycling, although its impact resistance decreases slightly, the tensile strength, elongation and hardness of ABS are constant (Kim and Kang, 1995).

Further all open source hardware-based equipment (Ackerman, 2008.; Gibb and Abadie, 2014) was used in all steps of the processing including an open source granulator (Appropedia, 2016a), a vertical recyclebot ac4.0 (Appropedia, 2016b), and delta-style RepRap (Appropedia, 2016c). Post-consumer ABS stabilizing feet (92.36 g /foot) for a 
Preprint: Shan Zhong \& Joshua M. Pearce. Tightening the loop on the circular economy: Coupled distributed recycling and manufacturing with recyclebot and RepRap 3-D printing,Resources, Conservation and Recycling 128, (2018), pp. 48-58. doi: 10.1016/j.resconrec.2017.09.023

5G tower or smart UPS as seen in Figure 1 was shredded by the granulator. The crushed plastic was used to make 3-D printing filament and then three case study consumer goods were manufactured including a camera tripod, an SD card holder and a camera hood. In order to compare this method with the combination of traditional recycling and traditional manufacture in energy consumption, the electricity consumed at each step was recorded by a multimeter (+/- $0.01 \mathrm{kWh})$. To account for mass loss at each processing step, at each stage of processing the plastic was massed with a digital balance (+/- $0.01 \mathrm{~g})$.

\subsection{Small-scale shredding of post-consumer plastic waste}

Mechanical cleaning of the post-consumer plastic waste is necessary before the shredding step. Impurities not only degrade overall filament consistency, but also increase the clogging frequency in the nozzle of the 3-D printer. Next, an open source plastic granulator/shredder was used to shred the plastic. To be more accessible to small and medium sized enterprises it was designed to operate on single phase power instead of three phase power, which is common for commercial industrial tools. The hopper of the granulator is designed to maintain mechanical integrity of the granulator so the entrance is $200 \mathrm{~cm}^{2}$ large to limit the size of the incoming plastic pieces. If the plastic particle is larger it will need to be reduced in size manually before depositing in the hopper to be crushed in the granulator chamber by spinning fly knives. Three fly knives rotate about an axis and striking a bed knife that is stationary on the outside of the path the rotary blades follow (Figure 2). The shaft and fly knife mounts are made out of A36 steel. The fly knives and bed knife are made out of tool steel, and the sieve is made out of stainless steel. As the plastic is granulated, it is sorted by a sieve, which has $1 / 8$ inch $(3.2 \mathrm{~mm})$ holes in it because that is the acceptable granulated size for the recyclebot. The particle size distribution was determined by imaging and the use of the open source imageJ software (imageJ, 2016).

ImageJ was also used to get the areas and perimeters of these filament cross sections. Based on these areas (a), and perimeters (p), the circularity, C, of filament can be calculated by:

$$
C=4 \pi\left(\frac{a}{p^{2}}\right)
$$

The circularity is a number with the range of $0-1$. $C=1$ indicates a perfect circle.

After shredding, the crushed plastic needs to be dried to maintain consistent quality by low temperature heating, or exposure to low-humidity environment by ambient, vacuum or desiccant. If this is not completed the moisture on the plastic vaporizes and form bubbles that roughen the filament surface. This can be seen in Figure 3, where the filament on the left was produce from moist pellets and the right one was from dried pellets. It is obvious that filament from dried particles has better quality.

\subsection{Extruding filament}

The full designs, build plans and operation of the recyclebot are maintained and updated at (Appropedia, 2016b). The two primary control parameters of the recyclebot are heating tube and extruder temperature and auger rotation speed. The temperature of heating tube should be above the glass transition temperature of the polymer being processed so that the polymer molecules have mobility, and below the decomposition temperature to avoid breaking of molecule structures. The glass transition temperature and decomposition temperature of ABS are $115.5^{\circ} \mathrm{C}$ and $300-450{ }^{\circ} \mathrm{C}$ respectively ( $\mathrm{Li}$ and Hiroshi, 2009; Wang et al., 2003), and the glass transition temperature of ABS is not 
Preprint: Shan Zhong \& Joshua M. Pearce. Tightening the loop on the circular economy: Coupled distributed recycling and manufacturing with recyclebot and RepRap 3-D printing,Resources, Conservation and Recycling 128, (2018), pp. 48-58. doi: 10.1016/j.resconrec.2017.09.023

changed after recycling while the decomposition temperature of ABS just increases slight by $3{ }^{\circ} \mathrm{C}$ (Kim \& Kang, 1995). While in this appropriate range, the molecule mobility would increase as temperature increases. The heating tube temperature was set as $180{ }^{\circ} \mathrm{C}$ here. The auger rotation speed determines the extrusion rate $(\mathrm{kg} / \mathrm{h})$ because the only plastic inputs into the tube is provided by the auger. It is better to set high rotation speed to get higher extrusion rates and avoid plastic staying in the heating tube for long time, which is important for filament quality. Long exposure times to high temperatures usually leads to the degradation of polybutadiene chain in ABS and affects the mechanical properties negatively, such as the decreasing of compact resistance (Tiganis et al., 2002; Blom et al., 2006). The auger rotation speed was set as 15 revolutions per min.

Filament diameter depends on the nozzle size and the tension on the filament after extruding. When the filament comes out through the nozzle, it swells slightly. Then as the tension increases, the filament diameter decreases. The recyclebot ac4.0 has a vertical geometry, as shown in Figure 4, so it uses gravity directly to provide tension on the filament after extruding. This is unlike previous studies on recyclebots and most commercial systems that use a horizontal geometry. At the beginning of extruding, the filament descended gradually and then was pulled through the light sensor, diameter measurement, length measurement, guide tube and then wound on the spooler. In this collecting system, there are two modes to collect the filament, the auto mode and the manual mode. In auto mode, when the filament descends and passes through the light sensor, the spooler begins to rotate and collect filament. If the rotation speed of spooler is too fast, the filament rises up and passed through the light sensor again, then the spooler slows and stops rotating. On manual mode, the rotation speed of spooler is adjusted manually by rotating a knob that controls the spooler speed on a panel. The manual mode is always used when the extrusion rate is not stable, such as the initial state of extrusion. To get a uniform size of filament, the filament should descend the same distance to keep the tension on the filament constant. To get the filament with 1.75 mm diameter, which was needed by the MOST delta-style RepRap, the nozzle size is $1.2 \mathrm{~mm}$ and the filament loop descended $30 \mathrm{~cm}$.

During heating and extruding process, the electricity and time consumed were collected along with the mass to calculate the extrusion rate and energy consumption. The filament diameter distribution was determined by imaging and the use of the open source imageJ software (imageJ, 2016).

\subsection{Printing Case Study Consumer Goods}

The Reprap used in this project is the Athena 3-D printer which derived from the MOST delta RepRap 3-D printer. The Athena is easier to assemble and maintain and has better wire management and flexibility, which increases its applicability for households. The basic RepRap printer was modified with a heated bed to ensure adhesion of ABS to the glass substrate (although other methods using compatible polymer print surfaces that enable bed adhesion without heating can be used). It should be noted, however, that a heated printing surface is preferred, as ABS will contract when cooled leading to warped parts. During large prints with ABS without a printed bed warping can cause delamination in between printed layers. ABS printing temperature is in the range of $200-240^{\circ} \mathrm{C}$. If the temperature is too high, the extruder would leak between the separate parts and cause stringing. If the temperature is too low, the plastic cannot stick well to the previous layer causing weak interlayer adhesion and under extrusion, which results in the printed part having reduced mechanical strength. 
Preprint: Shan Zhong \& Joshua M. Pearce. Tightening the loop on the circular economy: Coupled distributed recycling and manufacturing with recyclebot and RepRap 3-D printing,Resources, Conservation and Recycling 128, (2018), pp. 48-58. doi: 10.1016/j.resconrec.2017.09.023

\subsection{Case Study Objects}

The recycled ABS filament was used to test printing a camera tripod, SD card holder and camera hood. The camera bubble tripod consists of a tripod top, a tripod base, nine leg components and three tripod feet (Wilson, 2015). The SD card holder consists of a top side, bottom side, SD card container, micro-SD card container, card reader container and two multi-card containers (Drano, 2015). The camera hood fits a Canon 18-135 STM lens (Andrade, 2012).

The printing temperature was set as $220^{\circ} \mathrm{C}$ except for the tripod feet. When the tripod feet were being printed, the printing temperature was set as $220^{\circ} \mathrm{C}$ for first layer and $200{ }^{\circ} \mathrm{C}$ for other layers. The tripod foot has a relatively large sphere and one side always warped during printing, so decreasing the printing temperature was used to eliminate the issue. The heated bed temperature was set as $110{ }^{\circ} \mathrm{C}$ for all components. The printing speed was $60 \mathrm{~mm} / \mathrm{s}$, fan power at $80 \%$ and fill density was $100 \%$.

\section{Results}

\subsection{Shredding Post-Consumer Waste Plastic}

The open source granulator was successfully used to shred post-consumer ABS as shown in Figure 5. The energy consumption for shredding $1 \mathrm{~kg}$ of ABS is $0.138 \mathrm{kWh}$ and shredding rate is $4.358 \mathrm{~kg} / \mathrm{h}$. The shredded plastic was put into a vacuum chamber for half an hour. The vacuum chamber used in this study can contain about $1 \mathrm{~kg}$ of crushed plastic and it consumed $0.19 \mathrm{kWh}$ for 33 minutes vacuuming. The vacuuming rate is $1.818 \mathrm{~kg} / \mathrm{h}$.

The particle size distribution is important as it relates directly to the ease with which a recyclebot can produce uniform filament. The average particle size of the ABS pieces is $2.72 \mathrm{~mm}$, which is based on Figure 6 and Image J analysis. Figure 6 is a picture of a handful of ABS particles, which was ran through ImageJ to get each particle's top side area assuming each particle is a sphere. It should be noted that using this method to estimate particle size always gets a larger number compared to the real size if the pellets are not perfect spheres. Figure 7 is the particle size distribution of that handful of pellets. It is found that most particles are within the particle size range of 0.08-1.06 mm. In order to get the plastic quantity distribution with respect to the particle size, the top side area was used to compare the relative mass of each pellet. Figure 8 is the total top side areas of different particle size ranges. It is obvious that though these tiny pellets are the greatest in number their contribution to the total mass is negligible. Most of postconsumer ABS were shredded into the pieces with the particle size of 2.04-5.96 mm, and the amount of the particles within this range is more than half of the total amount. However, there are a few particles that are larger than $8 \mathrm{~mm}$ and need to be removed manually or by a sieve to make sure these crushed ABS are small enough to be used as feedstock for the recyclebot.

In addition, not all of the plastic fed into the granulator can be shredded and collected in the collector. There is always some plastic leftover between the chamber bottom and blades which cannot be shredded and get through the sieve, which generates the difference between the amount of post-consumer plastic and the amount of plastic particles. However, as more plastic waste is shredded, the influence of the difference is reduced.

\subsection{Extruding Filament}

The open source vertical recyclebot ac4.0 was used to make filament from crushed ABS. Before the extrusion process, it took 7 minutes and $0.005 \mathrm{kWh}$ for the heating tube to reach operation temperature. When the temperature rises up to the set point, the 
Preprint: Shan Zhong \& Joshua M. Pearce. Tightening the loop on the circular economy: Coupled distributed recycling and manufacturing with recyclebot and RepRap 3-D printing,Resources, Conservation and Recycling 128, (2018), pp. 48-58. doi: 10.1016/j.resconrec.2017.09.023

auger begins to rotate and filament is extruded. The initial 0.5 meters of filament was discarded because of inconsistency as the entire hot zone reached the set point temperature. The extrusion rate was $0.262 \mathrm{~kg} / \mathrm{h}$ and energy consumption for extruding $1 \mathrm{~kg}$ of filament is $0.302 \mathrm{kWh}$. This results in a single vertical recylebot capable of producing $6 \mathrm{~kg}$ of filament in a 24 hour day or a $1 \mathrm{~kg}$ spool in less than 4 hours. Figure 9 is a picture of recycled filament from the crushed ABS material. Compared to extrusion, the energy consumption for warming up the recyclebot is negligible if large quantities of filament is produced.

Figure 10 shows the filament cross sections from different parts of the spool. A knife was used to cut the filament perpendicular to its length in order to examine cross sections. ImageJ was used with Equation (1) to find that the circularity range of these cross sections is $0.81-0.89$ and the average circularity is 0.87 . This means the filament produced by recyclebot from crushed post-consumer ABS has good roundness and could be 3-D printed.

Figure 11 shows the distribution of filament diameters. The average diameter is $1.84 \mathrm{~mm}$, which was calculated from cross section areas. It is clear that the tolerance of this filament is $+0.1 /-0.04 \mathrm{~mm}$. The average diameter of commercial filament is 1.75 $\mathrm{mm}$ with tolerance of $+/-0.05 \mathrm{~mm}$. Compared to commercial filament, this recycled filament is slightly larger. Although the recyclebot settings could be tuned to more closely match commercial specifications, it is not necessary as the filament was well within the tolerances of the RepRap used for 3-D printing.

\subsection{Printing case study objects}

The open source Athena RepRap was used to test print recycled ABS filament. It took 8 minutes and $0.03 \mathrm{kWh}$ for the heated bed and nozzle to warm up. The components were printed one by one and the printed camera tripod, SD card holder and camera hood are shown in Figures 12-14, respectively.

\subsubsection{Camera tripod}

The energy consumption, time consumption and filament consumption of printing a camera bubble tripod were shown in Table 1. Including the heating process, printing a camera tripod needs $1.56 \mathrm{kWh}$ and 574 minutes (about 9.5 hours). During the whole printing process the energy consumption for initial heating is also small compared to the energy consumption for printing itself.

The total weight of 3-D printed camera tripod is 88.76 grams. The energy and time consumption for each step are summarized in Table 2. Based on the energy consumptions of $0.138 \mathrm{kWh} / \mathrm{kg}$ for shredding, $0.190 \mathrm{kWh} / \mathrm{kg}$ for vacuuming and 0.302 $\mathrm{kWh} / \mathrm{kg}$ for filament extruding, $88.76 \mathrm{~g}$ of plastic requires $0.012 \mathrm{kWh}$ for shredding, $0.017 \mathrm{kWh}$ for vacuuming and $0.027 \mathrm{kWh}$ for extruding filament. Based on the shredding rates of $4.358 \mathrm{~kg} / \mathrm{h}$, vacuuming rate of $1.818 \mathrm{~kg} / \mathrm{h}$ and extrusion rate of 0.262 $\mathrm{kg} / \mathrm{h}$, it takes 0.020 hours to shred $0.121 \mathrm{~kg}$ of plastic, 0.049 hours to vacuum and 0.339 hours to extrude it. Therefore, to produce a camera bubble tripod from post-consumer ABS, 9.975 hours and $1.616 \mathrm{kWh}$ were consumed in total.

\subsubsection{SD card holder}

The energy consumption, time consumption and filament consumption for 3-D printing a SD card holder are shown in Table 3. Including the heating process, printing a SD card holder needs $0.64 \mathrm{kWh}$ and 289 minutes in total.

The total weight of 3-D printed SD card holder is 77.6 grams. Table 4 summarizes the energy and time consumption for each step. Based on the energy 
Preprint: Shan Zhong \& Joshua M. Pearce. Tightening the loop on the circular economy: Coupled distributed recycling and manufacturing with recyclebot and RepRap 3-D printing,Resources, Conservation and Recycling 128, (2018), pp. 48-58. doi: 10.1016/j.resconrec.2017.09.023

consumptions of $0.138 \mathrm{kWh} / \mathrm{kg}$ for shredding, $0.190 \mathrm{kWh} / \mathrm{kg}$ for vacuuming and 0.302 $\mathrm{kWh} / \mathrm{kg}$ for filament extruding, $77.60 \mathrm{~g}$ of plastic requires $0.011 \mathrm{kWh}$ for shredding, $0.015 \mathrm{kWh}$ for vacuuming and $0.023 \mathrm{kWh}$ for extruding filament. Based on the shredding rates of $4.358 \mathrm{~kg} / \mathrm{h}$, vacuuming rate of $1.818 \mathrm{~kg} / \mathrm{h}$ and extrusion rate of 0.262 $\mathrm{kg} / \mathrm{h}$, it takes 0.018 hours to shred $77.60 \mathrm{~g}$ of plastic, 0.043 hours to vacuum and 0.296 hours to extrude it. Therefore, to produce a SD card holder from post-consumer ABS, 5.174 hours and $0.689 \mathrm{kWh}$ were consumed in total.

\subsubsection{Camera hood}

To 3-D print the camera hood $0.18 \mathrm{kWh}$ of electricity was consumed over 72 minutes using $17.37 \mathrm{~g}$ of recycled ABS filament. Including the heating process, printing a camera hood needs $0.21 \mathrm{kWh}$ and 80 minutes in total.

The total weight of 3-D printed camera hood is 17.37 grams. Based on the energy consumptions of $0.138 \mathrm{kWh} / \mathrm{kg}$ for shredding, $0.190 \mathrm{kWh} / \mathrm{kg}$ for vacuuming and $0.302 \mathrm{kWh} / \mathrm{kg}$ for filament extruding, $17.37 \mathrm{~g}$ of plastic requires $0.002 \mathrm{kWh}$ for shredding, $0.003 \mathrm{kWh}$ for vacuuming and $0.005 \mathrm{kWh}$ for extruding filament. Based on the shredding rates of $4.358 \mathrm{~kg} / \mathrm{h}$, vacuuming rate of $1.818 \mathrm{~kg} / \mathrm{h}$ and extrusion rate of $0.262 \mathrm{~kg} / \mathrm{h}$, it takes 0.004 hours to shred $17.37 \mathrm{~g}$ of plastic, 0.010 hours to vacuum and 0.066 hours to extrude it. Therefore, to produce a camera hood from post-consumer ABS, 1.410 hours and $0.220 \mathrm{kWh}$ were consumed in total.

\section{Discussion}

\subsection{Energy Savings}

This distributed recycling and production method with a recyclebot and RepRap saves energy not only in recycling process, but also in manufacturing process, which combine to improve the environmental performance in a circular economy. The energy consumption for ABS recycling in this project is $0.63 \mathrm{kWh} / \mathrm{kg}$ which is the sum of energy used in shredding, vacuuming and extruding filament process. The traditional plastic recycling method involves collecting and transporting post-consumer plastics to a collection center for separation, and then to reclamation facility for reconstruction. In a reclamation center, sorted plastic usually needs to be cleaned, dried, melted, extruded and then shredded into pellets for reuse (Barton ta al., 1996). This process is similar to the recycling process in this project, so it is assumed that their energy consumptions are nearly identical. This assumption is grounded in more detailed studies investigating recycling a different post-consumer plastic (HDPE), which found such results (Kreiger and Pearce, 2013a;b). But the traditional recycling method still requires large quantities of extra energy for transportation and sorting and compacting in the collection centers. The average energy consumption for transporting post-consumer plastics is 0.089 $\mathrm{kWh} / \mathrm{kg}$, for sorting is $0.075 \mathrm{kWh} / \mathrm{kg}$, for compacting is $0.025 \mathrm{kWh} / \mathrm{kg}$ (Arena et al., 2003), which means the traditional recycling method consumes an extra $0.189 \mathrm{kWh} / \mathrm{kg}$ compared to the distributed recycling method introduced in this project. Besides, the energy consumption for transportation would increase as the distance increases. Collecting waste plastics in remote rural areas usually requires much more energy (Kreiger et al., 2013a;b).

Figure 15 summarizes the energy consumption for producing the three case study products: a camera tripod, SD card holder and camera hood by traditional and distributed methods. The camera tripod, SD card holder and camera hood produced in the case study consumed $1.616 \mathrm{kWh}, 0.689 \mathrm{kWh}, 0.220 \mathrm{kWh}$ and their mass are 88.76 g, $77.60 \mathrm{~g}, 17.37 \mathrm{~g}$, respectively. The embodied energy of ABS is $36.667 \mathrm{kWh} / \mathrm{kg}$ which includes $13.500 \mathrm{kWh} / \mathrm{kg}$ feedstock energy (Wötzel et al., 1999; Hammond et al., 
Preprint: Shan Zhong \& Joshua M. Pearce. Tightening the loop on the circular economy: Coupled distributed recycling and manufacturing with recyclebot and RepRap 3-D printing,Resources, Conservation and Recycling 128, (2018), pp. 48-58. doi: 10.1016/j.resconrec.2017.09.023

2008). Except for the feedstock energy, $23.167 \mathrm{kWh}$ is needed to complete mining, processing natural resources, manufacturing and delivery to get $1 \mathrm{~kg}$ of ABS product. Therefore, the traditional manufacture method needs to consume $2.056 \mathrm{kWh}, 1.798$ $\mathrm{kWh}$ and $0.402 \mathrm{kWh}$ to produce a same camera tripod, SD card holder and camera hood, respectively based on their mass. If the feedstock energy is considered, extra raw material of $1.198 \mathrm{kWh}, 1.048 \mathrm{kWh}$ and $0.234 \mathrm{kWh}$ are required to produce ABS products of $88.76 \mathrm{~g}, 77.60 \mathrm{~g}$ and $17.37 \mathrm{~g}$ respectively. The percent of energy saved by the distributed process depends on the complexity of the objects with the tripod being the most complex and the camera hood being the least complex. From Figure 15, it is clear that using traditional manufacturing method to produce an ABS product consumes more than double the energy compared to coupled distributed recycling and manufacturing method. If the products of simple structures are produced, such as the case study products shown here, more energy can be conserved by this coupled distributed method.

As the energy consumption is less when prosumers (producing consumers) use distributed recycling and manufacturing methods, they can also reduce the greenhouse gas emissions to produce a product. The coupled distributed recycling and manufacturing method does not require post-consumer plastic transportation and product delivery, so it directly decreases carbon emissions from the combustion of transportation-related fuel. In addition, distributed recycling and manufacturing consumes less energy than the traditional recycling process and manufacturing process, so carbon emission decreases further from this conserved energy. This again improves the environmental performance of the circular economy. According to the energy conservation and carbon emission mitigation, it is clear that this method has great benefit for environment and supports a growing body of evidence in this regard (Kohtala, 2015).

\subsection{Economics}

Regardless of the environmental benefits of the circular economy as a whole or of the distributed approach to recycling and/or manufacturing discussed here, they are unlikely to be widely adopted by consumers without a significant financial incentive. Fortunately, as the results of this study show, there is such an incentive. The total energy consumption for producing a camera bubble tripod, SD card holder and camera hood in this project is $1.616 \mathrm{kWh}, 0.689 \mathrm{kWh}$ and $0.220 \mathrm{kWh}$ respectively, so their cost can be estimated as 19 cents, 8 cents and 3 cents based on the average electricity price in U.S. which is US $\$ 0.12 / \mathrm{kWh}$. This represents substantial savings for consumer products. For example, the lowest-cost equivalent on Amazon for the similar camera bubble tripod costs US\$3.49 dollars (Amazon, 2016a) instead of 19 cents. Similarly, an equivalent camera lens hood costs US\$9.99 dollars (Amazon, 2016b) while the 3-D printed from recycled ABS waste costs only 3 cents. Thus, one could make 333 camera lens hoods for the same economic cost as purchasing a single one by conventional distributors. Although the commons-based SD card holder design here is new and there is no identical commercial one, a similar one costs US\$5.98 (Amazon, 2016c) instead of 8 cents to make at home from waste platic.

The economic benefit of distributed recycling and manufacture method is obvious and even greater than those found earlier for creative commons designs coupled with commercial filament and 3-D printers (Wittbrodt, et al, 2013; Petersen and Pearce, 2017). This is for only simple products. Products with complex structures are always even relatively more expensive because of the restrictions in traditional manufacture methods. However, the complex structure does not make much difference in cost in the 
Preprint: Shan Zhong \& Joshua M. Pearce. Tightening the loop on the circular economy: Coupled distributed recycling and manufacturing with recyclebot and RepRap 3-D printing,Resources, Conservation and Recycling 128, (2018), pp. 48-58. doi: 10.1016/j.resconrec.2017.09.023

3-D printing method, which is why for example sophisticated scientific equipment can be produced for $1 \%$ of the cost of those tools made by traditional methods (Pearce, 2012;2013). In addition, as using this method allows products to be produced at home and do not need the transportation and associated shipping costs the cost of the product can be reduced further. Again, these costs add no value to the product and only have a negative environmental impact.

The low cost and high quality are always the most important standards for people to evaluate a product. The latter is a challenge as it is known that with each recycling pass polymer mechanical properties degrade (Sanchez et al., 2017), although the filament quality remains acceptable for most consumer goods through five cycles (Sanchez et al., 2015). For the products investigated here as case studies the mechanical strength loss is minimal and the materials are more than adequate for these applications. In addition, if necessary a smoothing of the ABS can be accomplished with acetone to obtain commercial quality aesthetics (Winbrodt et al., 2013). There is no doubt that coupled recycling and manufacturing methods with large economic benefit is able to encourage people to recycle more plastic and produce products by themselves. There is already considerable evidence that this is happening at least for distributed manufacturing in some markets (Petersen et al., 2017). To see how substantial this can be and why this is occurring, consider the lens hood: When the $92.36 \mathrm{~g}$ stabilizing foot is either broken or not needed, it can be recycled by coupled distributed method and almost 5 camera lens hood can be produced from it, which means the value of approximately US $\$ 50.00$ is created from one stabilizing foot.

In contrast, the post-consumer plastic bottle recycle rate in America in 2013 is 30.9\% (Plastic American-Chemistry, 2013), which results in about two thirds of plastic bottles being disposed by incineration and landfill. These recycled bottles are pure materials such as PET and HDPE and also be separated and recycled using the methods discussed here for ABS e-waste. However, those plastics that are used in electronic product, such as ABS, are always coupled to other materials so they usually have relatively lower recycle rates. With the stimulation for a circular economy created by the economic benefit, the post-consumer plastic recycle rate would potentially increase as the tools become more widely available for people to recycle more plastics in their homes or communities.

Finally, the cost of a recyclebot must be factored into the economics of the use of recycled filament. This cost is currently well under 1000 USD with several recyclebotderivative kits available on the web for a few hundred USD. This cost can thus be justified with the creation of approximately $5 \mathrm{~kg}$ of filament per 100USD of filament savings. Depending upon the frequency of use of the 3-D printer the recyclebot may thus be economically appropriate for individual homes. Particularly if coupled with the 3-D printer (which already has a well-established rapid payback time and high return on investment (ROI) (Petersen \& Pearce, 2017)) it is clear the combination would also provide a high ROI. However, for low-usage homes it would be better scaled in a community center (e.g. library, makerspace, fablab, or small business) where many families (e.g. using only a few kg/year) could benefit from it. Future work is necessary to gauge average usage rates among 3-D printer operators making products for household use. In addition, in the future, more advanced polymers and composites (Tian, et al., 2016) can be explored in this low-cost open source distributed upcycling case for a circular economy.

\subsection{Implications of Direct Digital Manufacturing on the Circular Economy}


Preprint: Shan Zhong \& Joshua M. Pearce. Tightening the loop on the circular economy: Coupled distributed recycling and manufacturing with recyclebot and RepRap 3-D printing,Resources, Conservation and Recycling 128, (2018), pp. 48-58. doi: 10.1016/j.resconrec.2017.09.023

These results take the digital manufacturing optimization (Agustí-Juan and Habert, 2017) and direct digital manufacturing (Chen et al., 2015) to the extreme case discussed by Kostakis et al. (2017). Kostakis et al. focus is on the model of designing globally, but manufacturing locally and builds on the conjunction of the digital commons of knowledge and design (e.g. the three commons-based designs used for case studies here) with desktop and bench-top manufacturing technologies (such as the open source 3-D printers used in this study). It is clear from the results of the study reported here that in the short-to-medium term waste plastic from discarded e-waste can be significantly upcycled using this commons-based approach for the benefit of the environment as well as the economic stability of consumers. This coupled distributed recycling and manufacturing method helps to solve post-consumer plastic disposal issues and raw material shortage issues as it uses recycled plastic to produce products. This method closes the loop of plastic material flows, which not only assists the circular economy as a whole, but also the sustainability at the household level. In addition, it improves upon the standard view of the circular economy occurring at the industrial level with significant transport between stages of a products life cycle. In this case the transport loop is significantly tightened, literally turning waste into products in the home of the consumer.

Distributed manufacturing with 3-D printing also directly benefits the goals of the circular economy. When the 3-D printed part is no longer useful, it can be shredded and used to make filament and printed into new useful products. It is interesting to note that a broken stabilizer foot (the raw material used for this study) could be recycled using this method into a new stabilizing foot. Each foot weighs less than $93 \mathrm{~g}$ (and it could be printed with lower infill to weigh even less). In addition, the shipping weight of a single foot is $1 \mathrm{lb}$ (453g), which is a factor of 4.87x due presumably to the mass of the packaging to ship one (Amazon, 2016d). The stabilizing foot costs US\$9.99 on Ebay (E-bay, 2016), which again shows considerable economic savings using the coupled distributed recycling-manufacturing process.

\subsection{Future Work}

More work is needed in this area to determine how many cycles are technically feasible following Sanchez et al. (2015). For example, the thermal properties would change and torsion strength would decrease as more recycled ABS is being mixed in the virgin ABS resin (Chen et al., 2011). Future work is necessary to investigate the difference of thermal properties and physical properties between printed parts from recycled $A B S$ filament and virgin $A B S$ filament, and try to remedy the degradation influence of multiple cycles.

Lastly, the printing process consumed more than $90 \%$ of the whole energy for both processes. This is because printing with a heated bed consumes large quantities of energy to maintain the bed at $110^{\circ} \mathrm{C}$. Future work is necessary to improve the printing method to decrease the energy consumption with printing with ABS. Melted ABS cannot stick on the cold glass, but it can stick well on PLA thin layers. If the ABS is printed on the PLA thin layer instead of heated bed, more than half of the energy can be saved. Besides, if the whole system is powered by solar photovoltaic panels (Gwamuri et al., 2016) instead of tradition electricity grid, the energy used can also be totally conserved, which significantly improves environmental performance (Zhong, et al, 2017).

Lastly, and most importantly, a new blueprint for the circular economy must be constructed using distributed manufacturing with 3-D printers and the use of recyclebots to aid in distributed recycling on a large scale. The benefits (e.g. less embodied energy 
Preprint: Shan Zhong \& Joshua M. Pearce. Tightening the loop on the circular economy: Coupled distributed recycling and manufacturing with recyclebot and RepRap 3-D printing,Resources, Conservation and Recycling 128, (2018), pp. 48-58. doi: 10.1016/j.resconrec.2017.09.023

of transportation, and packaging) must be quantified when a significant penetration of distributed manufacturing is present. At the same time, material limitations as outlined above must be taken into account in order to capture realistic environmental impacts from for example the need to use some virgin polymer materials to maintain mechanical integrity of thermoplastics making multiple recycle loops.

\section{Conclusions}

A circular economy is an industrial economy that promotes greater resource productivity aiming to reduce waste and avoid pollution by design in which material flows of technical nutrients are recycled in the industrial system. This study has shown that the circle can be tightened by bringing the industrial system within in a single home, business, or community center. In this tight circle, the value can be generated continually as post-consumer products are used to produce new products. In addition, when the materials flow in this circle, less energy is consumed and less greenhouse gas is emitted than would be otherwise to meet the same consumer desire than used either in a traditional circular economy paradigm or a standard centrally-manufactured paradigm. This study presented a distributed recycling and manufacturing method with the coupling of an open source recyclebot and RepRap 3-D printer. Post-consumer ABS was recycled and then used as material to produce three case study products. From the three case studies, it is clear that using traditional manufacturing methods to produce an ABS product consumes more than double the energy compared to coupled distributed recycling and manufacturing method.

Therefore, this couple distributed recycling and manufacturing method fits well into the goal of circular economy and meets the requirement of sustainable development.

Finally, the economic analysis presented here indicates that the economic benefit to consumers can be used to encourage adoption of circular economic practices. By tightening the recycling and production loops the circular economy is supported by this method.

\section{References}

Ackerman J.R. Toward open source hardware. U. Dayton L. Rev. 2008;34:183.

Amazon. 2016a. https://www.amazon.com/Flexible-Bendable-SSE-Microfiber-

Cleaning/dp/B00K55IMS2 (visited 12.18.2016)

Amazon. 2016b. https://www.amazon.com/Replacement-Altura-Photo-18-135mm-3-55-6/dp/B0045FLMIA (visited 12.18.2016)

Amazon. 2016c. https://www.amazon.com/Slots-Holder-Memory-Card-memoryCarrying/dp/B00CGTMXNK (visited 12.18.2016)

Amazon. 2016d. APC 0J-876-0011A PLASTIC STABILIZING FOOT 5G TOWER ROHS https://www.amazon.com/0J-876-0011A-PLASTIC-STABILIZING-FOOTTOWER/dp/B00DEMM7KM (visited 12.26.2016).

Andersen M.S. An introductory note on the environmental economics of the circular economy. Sustainability Science 2007;2(1):133-140.

Andrade, L. 2012. Canon 18-135 STM lens hood. Available: http://www.thingiverse.com/thing:38336/ (visited 12.10.2016)

Anzalone G.C., Wijnen, B. and Pearce, J.M. Multi-material additive and subtractive prosumer digital fabrication with a free and open-source convertible delta RepRap 3-D printer. Rapid Prototyping Journal 2015;21(5):506-519.

Appropedia. Open Source Hardware Enterprise Plastic Granulator. 2016a. http://www.appropedia.org/Open_Source_Hardware_Enterprise_Plastic_Granulator (visited 12.8.2016) 
Preprint: Shan Zhong \& Joshua M. Pearce. Tightening the loop on the circular economy: Coupled distributed recycling and manufacturing with recyclebot and RepRap 3-D printing,Resources, Conservation and Recycling 128, (2018), pp. 48-58. doi: 10.1016/j.resconrec.2017.09.023

Appropedia. Recyclebot 2016b. http://www.appropedia.org/Recyclebot (visited 9.8.2016)

Appropedia. Athena Build Overivew. 2016c. http://www.appropedia.org/Athena_Build_Overview (visited 9.8.2016)

Arena U., Mastellone M.L. and Perugini F. Life cycle assessment of a plastic packaging recycling system. The international journal of life cycle assessment 2003;8(2):9298.

Astrup, T., Møller, J. and Fruergaard, T. Incineration and co-combustion of waste: accounting of greenhouse gases and global warming contributions. Waste Management \& Research 2009;27(8):789-799.

Agustí-Juan, I. and Habert, G. Environmental design guidelines for digital fabrication. Journal of Cleaner Production 2017;142(4):2780-2791.

Baich, L., Manogharan, G., Marie, H., Study of infill print design on production costtime of 3D printed ABS parts. International Journal of Rapid Manufacturing 2015; 5(3-4):308-319.

Baechler C., DeVuono M., and Pearce J.M. Distributed recycling of waste polymer into RepRap feedstock. Rapid Prototyping Journal 2013;19(2):118-125.

Barton J. R., D. Dalley, and V. S. Patel. Life cycle assessment for waste management. Waste management 1996;16(1):35-50.

Bicket M., Guilcher S., Hestin M., Hudson C., Razzini P., Tan A., Ten Brink P., Van Dijl E., Vanner R. and Watkins E., 2014. Scoping study to identify potential circular economy actions, priority sectors, material flows and value chains. European Commission; 2014.

Birtchnell, T., Urry, J., Cook, C. and Curry, A., 2013. Freight miles: the impact of 3D printing on transport and society.

Blom Henk, Rosa Yeh, Robert Wojnarowski, and Michael Ling. Detection of degradation of ABS materials via DSC. Journal of thermal analysis and calorimetry 2006;83(1):113-115.

Charter, M. and Keiller, S., 2014. Grassroots innovation and the circular economy: a global survey of repair cafés and hackerspaces.

Chen, D., Heyer, S., Ibbotson, S., Salonitis, K., Steingrímsson, J.G. and Thiede, S. Direct digital manufacturing: definition, evolution, and sustainability implications. Journal of Cleaner Production 2015;107:615-625.

Chen, S.C., Liao, W.H., Hsieh, M.W., Chien, R.D. and Lin, S.H. Influence of recycled ABS added to virgin polymers on the physical, mechanical properties and molding characteristics. Polymer-Plastics Technology and Engineering 2011; 50(3):306-311.

Craighill, A. L., and Powell, J.C. Lifecycle assessment and economic evaluation of recycling: a case study. Resources, Conservation and Recycling 1996;17(2):75-96.

Crane N. B., J. Tuckerman, and G. N. Nielson. Self-assembly in additive manufacturing: opportunities and obstacles. Rapid Prototyping Journal 2011;17(3):211-217.

Despeisse, M., Baumers, M., Brown, P., Charnley, F., Ford, S.J., Garmulewicz, A., Knowles, S., Minshall, T.H.W., Mortara, L., Reed-Tsochas, F.P. and Rowley, J., 2017. Unlocking value for a circular economy through 3D printing: a research agenda. Technological Forecasting and Social Change, 115, pp.75-84.

Drano. D. 2015. Swiss Army Style SD Holder (Expandable)(Raspberry adaptors). Available: http://www.thingiverse.com/thing:633436 (visited 12.10.2016)

E-bay. 2016. http://www.ebay.com/itm/APC-0J-876-0011A-PLASTIC-STABILIZINGFOOT-5G-TOWER-or-SMART-UPS-/162291598475(visited 12.19.2016).

Ford, S. and Despeisse, M. Additive manufacturing and sustainability: an exploratory 
Preprint: Shan Zhong \& Joshua M. Pearce. Tightening the loop on the circular economy: Coupled distributed recycling and manufacturing with recyclebot and RepRap 3-D printing,Resources, Conservation and Recycling 128, (2018), pp. 48-58. doi: 10.1016/j.resconrec.2017.09.023

study of the advantages and challenges. Journal of Cleaner Production 2016;137: 1573-1587

Gebhardt, A., Schmidt, F.M., Hötter, J.S., Sokalla, W. and Sokalla, P. Additive manufacturing by selective laser melting the realizer desktop machine and its application for the dental industry. Physics Procedia 2010;5:543-549.

Gertsakis, J., and Lewis, H. Sustainability and the waste management hierarchy. A discussion paper prepared for EcoRecycle Victoria, March 2003.

Gibb A. Building open source hardware: DIY manufacturing for hackers and makers. Pearson Education; 2014.

Gu, L. and Ozbakkaloglu, T. Use of recycled plastics in concrete: A critical review. Waste Management 2016;51:19-42.

Gorechi P. Schaub A., Wilder L. Ford college community challenge: Plastic GranulatorFinal report. Open Source Hardware Enterprise; 2016.

Gwamuri, J., Franco, D., Khan, K.Y., Gauchia, L. and Pearce, J.M. High-Efficiency Solar-Powered 3-D Printers for Sustainable Development. Machines 2016;4(1):3.

Hammond, G.,Jones, C., Lowrie F., and P. Tse. Inventory of carbon \& energy: ICE. Bath: Sustainable Energy Research Team, Department of Mechanical Engineering, University of Bath; 2008.

Hayami, Y., Dikshit, A.K. and Mishra, S.N. Waste pickers and collectors in Delhi: poverty and environment in an urban informal sector. The Journal of Development Studies 2006, 42(1):41-69.

Hunt, E. J., Zhang C, Anzalone N, Pearce JM. Polymer recycling codes for distributed manufacturing with 3-D printers. Resources, Conservation and Recycling 2015;97:24-30.

ImageJ. 2016. https://imagej.nih.gov/ij/ (visted Dec. 27, 2016).

Jacobsen, N.B. Industrial symbiosis in Kalundborg, Denmark: a quantitative assessment of economic and environmental aspects. Journal of Industrial Ecology 2006;10(12):239-255.

Jones, R., Haufe, P., Sells, E., Iravani, P., Olliver, V., Palmer, C. and Bowyer, A. RepRap-the replicating rapid prototyper. Robotica 2011;29(1):177-191.

Kietzmann J., Pitt L. and Berthon P. Disruptions, decisions, and destinations: Enter the age of 3-D printing and additive manufacturing. Business Horizons 2015;58(2):209215.

Kim Jin Kuk, and Chang K. Kang. Basic studies on recycling of ABS resin. PolymerPlastics Technology and Engineering 1995;34(6):875-890.

Kohtala, C. Addressing sustainability in research on distributed production: an integrated literature review. Journal of Cleaner Production 2015;106:654-668.

Kostakis, V., Latoufis, K., Liarokapis, M. and Bauwens, M., 2016. The convergence of digital commons with local manufacturing from a degrowth perspective: Two illustrative cases. Journal of Cleaner Production. (in press). http://dx.doi.org/10.1016/j.jclepro.2016.09.077

Kreiger M. A., M. L. Mulder, A. G. Glover, and Joshua M. Pearce. Life cycle analysis of distributed recycling of post-consumer high density polyethylene for 3-D printing filament. Journal of Cleaner Production 2014;70:90-96.

Kreiger M., Gerald C. Anzalone, M. L. Mulder, A. Glover, and Joshua M. Pearce. Distributed recycling of post-consumer plastic waste in rural areas. In MRS Proceedings, 1492:91-96. Cambridge University Press; 2013.

Kreiger, M., and Pearce, J.M. Environmental impacts of distributed manufacturing from 3-D printing of polymer components and products. In MRS Proceedings, 1492:8590. Cambridge University Press; 2013. 
Preprint: Shan Zhong \& Joshua M. Pearce. Tightening the loop on the circular economy: Coupled distributed recycling and manufacturing with recyclebot and RepRap 3-D printing,Resources, Conservation and Recycling 128, (2018), pp. 48-58. doi:

10.1016/j.resconrec.2017.09.023

Kreiger, M., and Pearce, J.M. Environmental life cycle analysis of distributed threedimensional printing and conventional manufacturing of polymer products. ACS Sustainable Chemistry \& Engineering 2013;1(12):1511-1519.

Li, Y. and Shimizu, H. Improvement in toughness of poly (l-lactide)(PLLA) through reactive blending with acrylonitrile-butadiene-styrene copolymer (ABS): Morphology and properties. European Polymer Journal 2009;45(3):738-746.

MacArthur, E. Towards the circular economy. Journal of Industrial Ecology 2013. Minnesota Office of Environmental Assistance. Recycling used electronics report on Minnesota's demonstration project; July 2001.

Mohr, S. and Khan, O., 2015. 3D printing and its disruptive impacts on supply chains of the future. Technology Innovation Management Review, 5(11), p.20.

Mota C. The rise of personal fabrication. In Proceedings of the 8th ACM conference on Creativity and cognition 2011;279-288. ACM.

Passamonti, F. J., and Sedran, U. Recycling of waste plastics into fuels. LDPE conversion in FCC. Applied Catalysis B: Environmental 2012;125:499-506.

Pearce, J.M., Blair, C.M., Laciak, K.J., Andrews, R., Nosrat, A. and Zelenika-Zovko, I. 3-D printing of open source appropriate technologies for self-directed sustainable development. Journal of Sustainable Development 2010;3(4):17.

Pearce, J.M. Building research equipment with free, open-source hardware. Science 2012;337(6100):1303-1304.

Pearce, J.M., 2013. Open-source lab: how to build your own hardware and reduce research costs. Elsevier.

Pearce J.M.. Applications of open source 3-D printing on small farms. Organic Farming 2015;1(1):19-35.

Petersen, E.E. and Pearce, J., 2017. Emergence of Home Manufacturing in the Developed World: Return on Investment for Open-Source 3-D Printers. Technologies, 5(1), 7; doi:10.3390/technologies5010007

Petersen, E.E., Kidd, R.W. and Pearce, J.M., 2017. Impact of DIY Home Manufacturing with 3D Printing on the Toy and Game Market. Technologies, 5(3), 45; doi:10.3390/technologies5030045

Plastic American-Chemistry. 2013. United States national post-consumer plastics bottle recycling report. https://plastics.americanchemistry.com/EducationResources/Publications/2013-National-Post-Consumer-Plastics-Bottle-RecyclingReport.pdf

Plastics Europe. Global plastic production from 1950 to 2015 (in million metric tons). https://www.statista.com/statistics/282732/global-production-of-plastics-since1950/, October, 2016.

Preston, F. A global redesign? Shaping the circular economy. Energy, Environment and Resource Governance 2012;2:1-20.

Redlich T. and Moritz M.. Bottom-up Economics. Foundations of a Theory of Distributed and Open Value. The Decentralized and Networked Future of Value Creation: 3D Printing and its Implications for Society, Industry, and Sustainable Development 2016:27.

Richardson M. and Haylock B. Designer/maker: the rise of additive manufacturing, domestic-scale production and the possible implications for the automotive industry. Computer-Aided Design \& Applications PACE 2012;2:33-48.

Ross, S. and Evans, D. The environmental effect of reusing and recycling a plasticbased packaging system. Journal of Cleaner Production 2003;11(5):561-571.

Sanchez, F.A.C., Lanza, S., Boudaoud, H., Hoppe, S. and Camargo, M., 2015, August. Polymer Recycling and Additive Manufacturing in an Open Source context: 
Preprint: Shan Zhong \& Joshua M. Pearce. Tightening the loop on the circular economy: Coupled distributed recycling and manufacturing with recyclebot and RepRap 3-D printing,Resources, Conservation and Recycling 128, (2018), pp. 48-58. doi: 10.1016/j.resconrec.2017.09.023

Optimization of processes and methods. In Annual International Solid Freeform Fabrication Symposium, ISSF 2015 (pp. 1591-1600).

Sanchez, F.A.C., Boudaoud, H., Hoppe, S. and Camargo, M., 2017. Polymer Recycling in an Open-Source Additive Manufacturing Context: Mechanical Issues. Additive Manufacturing. (in press).

Schenck, R. and Blaauw, P.F. The work and lives of street waste pickers in Pretoria-a case study of recycling in South Africa's urban informal economy. In Urban Forum 2011, 22(4):411-430).

Sells, E., Smith, Z., Bailard, S., Bowyer, A. and Olliver, V. RepRap: the replicating rapid prototyper: maximizing customizability by breeding the means of production. Handbook of Research in Mass Customization and Personalization, 2010.

Shen, L., Haufe, J. and Patel, M.K. Product overview and market projection of emerging bio-based plastics PRO-BIP 2009. Report for European Polysaccharide Network of Excellence (EPNOE) and European Bioplastics 2009:243.

Sinha, V., Patel, M.R. and Patel, J.V. PET waste management by chemical recycling: a review. Journal of Polymers and the Environment 2010;18(1):8-25.

Stahel, W.R., 2016. Circular economy: a new relationship with our goods and materials would save resources and energy and create local jobs. Nature, 531(7595), pp.435439.

Tansel, B. and Yildiz, B.S. Goal-based waste management strategy to reduce persistence of contaminants in leachate at municipal solid waste landfills. Environment, development and sustainability 2011;13(5):821-831.

Themelis N. J., M. J. Castaldi, J. Bhatti, and L. Arsova. Energy and economic value of non-recycled plastics (NRP) and municipal solid wastes (MSW) that are currently landfilled in the fifty states. New York, NY: Columbia University; 2011.

Tian, X., Liu, T., Wang, Q., Dilmurat, A., Li, D. and Ziegmann, G. Recycling and remanufacturing of 3D printed continuous carbon fiber reinforced PLA composites. Journal of Cleaner Production 2017; 142(4):1609-1618.

Tiganis B. E., L. S. Burn, Paul Davis, and A. J. Hill. Thermal degradation of acrylonitrile-butadiene-styrene (ABS) blends. Polymer degradation and stability 2002;76(3):425-434.

Van Wijk, A.J.M. and van Wijk, I., 2015. 3D Printing with biomaterials: Towards a sustainable and circular economy. IOS press.

Wang, S., Hu, Y., Wang, Z., Yong, T., Chen, Z. and Fan, W. Synthesis and characterization of polycarbonate/ABS/montmorillonite nanocomposites. Polymer Degradation and Stability 2003;80(1):157-161.

Webb, H.K., Arnott, J., Crawford, R.J. and Ivanova, E.P. Plastic degradation and its environmental implications with special reference to poly (ethylene terephthalate). Polymers 2012;5(1):1-18.

Wilson, J. 2015. Action Camera Flex Bubble Tripod. Available: http://www.thingiverse.com/thing:1201115 (visited 12.10.2016)

Wilson, D.C., Velis, C. and Cheeseman, C., Role of informal sector recycling in waste management in developing countries. Habitat International 2006, 30(4):797-808.

Wittbrodt B.T., Glover A.G., Laureto J., Anzalone G.C., Oppliger D., Irwin J.L. and Pearce J.M.. Life-cycle economic analysis of distributed manufacturing with opensource 3-D printers. Mechatronics 2013;23(6):713-726.

Wittbrodt B., Laureto J., Tymrak B. and Pearce, J.M.. Distributed manufacturing with 3D printing: a case study of recreational vehicle solar photovoltaic mounting systems. Journal of Frugal Innovation 2015;1(1):1-7.

Wittbrodt B.T. and Pearce J.M.. Total US cost evaluation of low-weight tension-based 
Preprint: Shan Zhong \& Joshua M. Pearce. Tightening the loop on the circular economy: Coupled distributed recycling and manufacturing with recyclebot and RepRap 3-D printing,Resources, Conservation and Recycling 128, (2018), pp. 48-58. doi: 10.1016/j.resconrec.2017.09.023

photovoltaic flat-roof mounted racking. Solar Energy 2015;117:89-98.

Wötzel K., R. Wirth, and M. Flake. Life cycle studies on hemp fibre reinforced components and ABS for automotive parts. Die Angewandte Makromolekulare Chemie 1999;272(1):121-127.

Yuan, Z., Bi, J. and Moriguichi, Y. The circular economy: A new development strategy in China. Journal of Industrial Ecology 2006;10(1-2):4-8.

Zhang, J., Wang, X., Gong, J. and Gu, Z. A study on the biodegradability of polyethylene terephthalate fiber and diethylene glycol terephthalate. Journal of Applied Polymer Science 2004;93(3):1089-1096.

Zhong, S., Rakhe, P. and Pearce, J.M., 2017. Energy Payback Time of a Solar Photovoltaic Powered Waste Plastic Recyclebot System. Recycling, 2(2), 10; doi: 10.3390/recycling2020010

Zhu D. J. Sustainable development calls for circular economy. Science and Technology Journal 1998;9:39-42.

Funding details. This study was supported by Tech for Trade and by Fulbright Finland. Disclosure statement. The authors have no conflict of interest.

Biographical note.

Shan Zhong is a graduate student in the Department of Materials Science \& Engineering at the Michigan Technological University.

Joshua M. Pearce is Professor cross-appointed in the Department of Materials Science \& Engineering and in the Department of Electrical \& Computer Engineering at the Michigan Technological University where he runs the Open Sustainability Technology Research Group. He is currently at Aalto University where he is the Fulbright-Aalto University Distinguished Chair. His research concentrates on the use of open source appropriate technology to find collaborative solutions to problems in sustainability and poverty reduction.

Geolocation information. U.S.A., Finland 
Preprint: Shan Zhong \& Joshua M. Pearce. Tightening the loop on the circular economy: Coupled distributed recycling and manufacturing with recyclebot and RepRap 3-D printing,Resources, Conservation and Recycling 128, (2018), pp. 48-58. doi: 10.1016/j.resconrec.2017.09.023

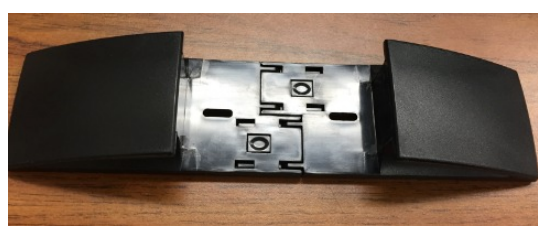


Preprint: Shan Zhong \& Joshua M. Pearce. Tightening the loop on the circular economy: Coupled distributed recycling and manufacturing with recyclebot and RepRap 3-D printing,Resources, Conservation and Recycling 128, (2018), pp. 48-58. doi: 10.1016/j.resconrec.2017.09.023

\section{Figure Captions}

Figure 1. Post-consumer stabilizing foot made of ABS.

Figure 2. Model of open source granulator v1 cutting chamber assembly.

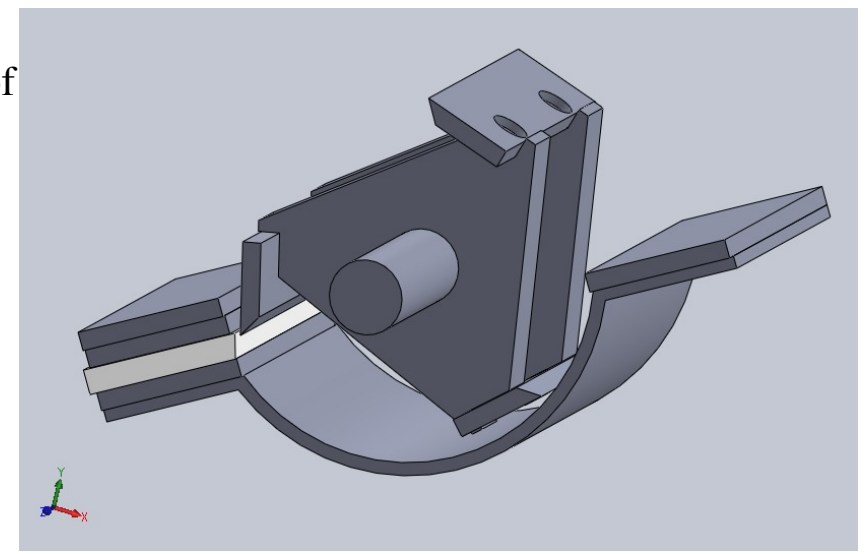

Figure 3.

Comparison of filament quality from moist pellets and dried pellets using identical process conditions in the recyclebot.

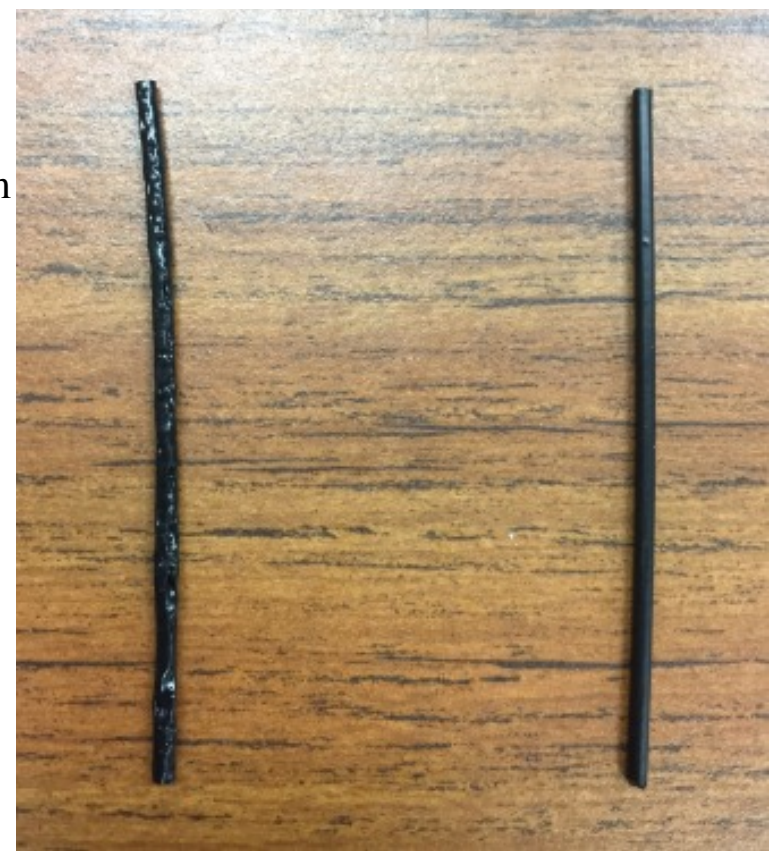


Preprint: Shan Zhong \& Joshua M. Pearce. Tightening the loop on the circular economy: Coupled distributed recycling and manufacturing with recyclebot and RepRap 3-D printing,Resources, Conservation and Recycling 128, (2018), pp. 48-58. doi: 10.1016/j.resconrec.2017.09.023

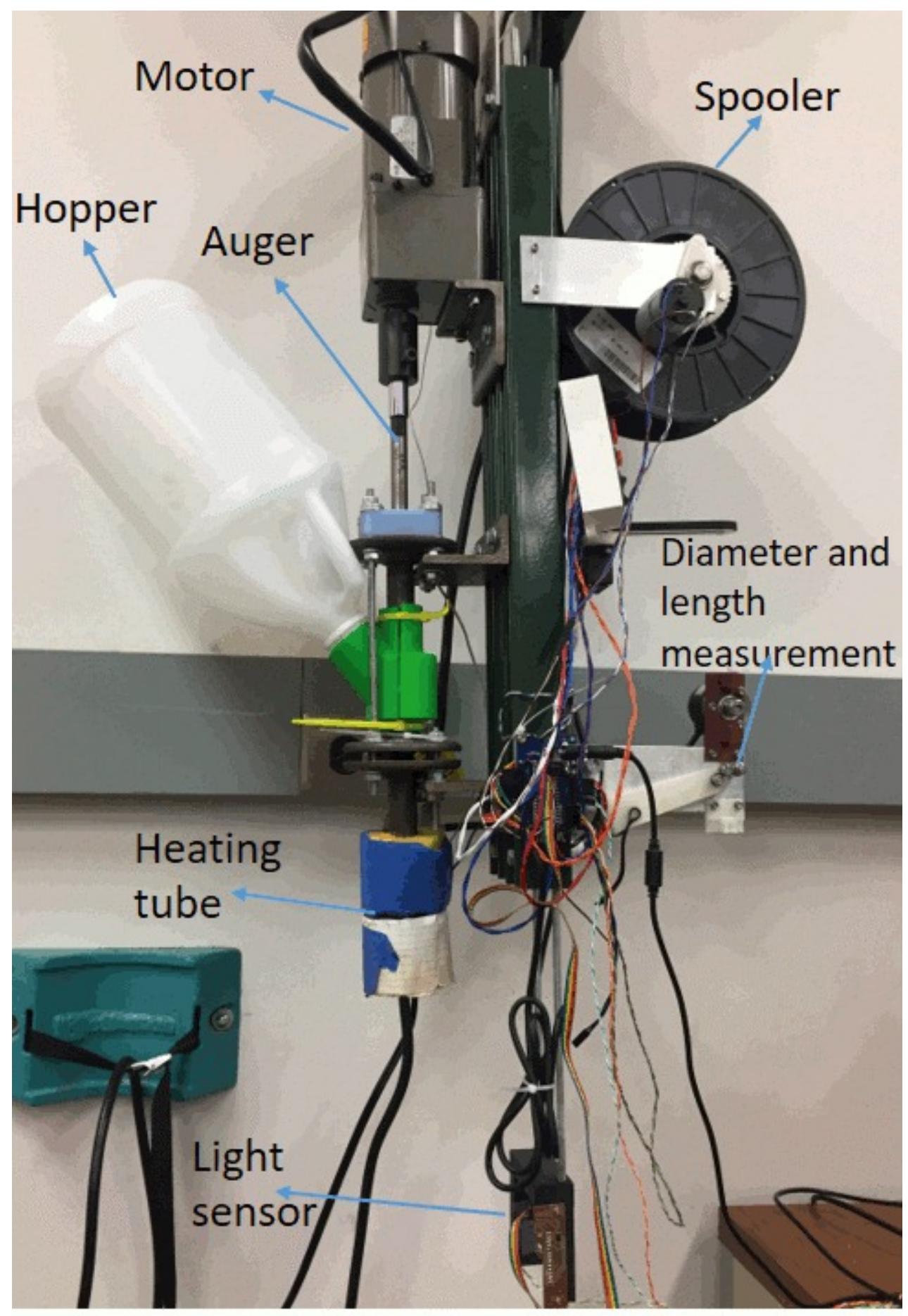


Preprint: Shan Zhong \& Joshua M. Pearce. Tightening the loop on the circular economy: Coupled distributed recycling and manufacturing with recyclebot and RepRap 3-D printing,Resources, Conservation and Recycling 128, (2018), pp. 48-58. doi: 10.1016/j.resconrec.2017.09.023

Figure 4. Recyclebot ac4.0.

Figure 5. The postconsumer ABS plastic and crushed ABS pellets

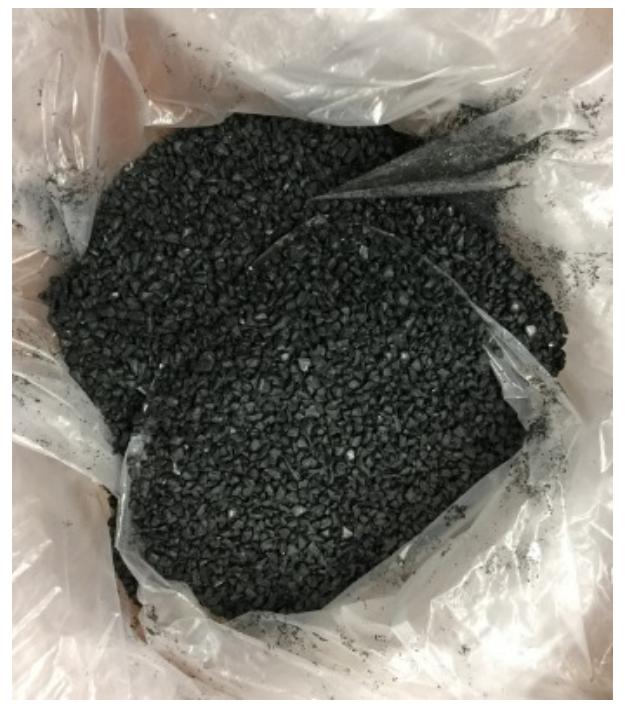

Figure 6. A handful of crushed postconsumer ABS.

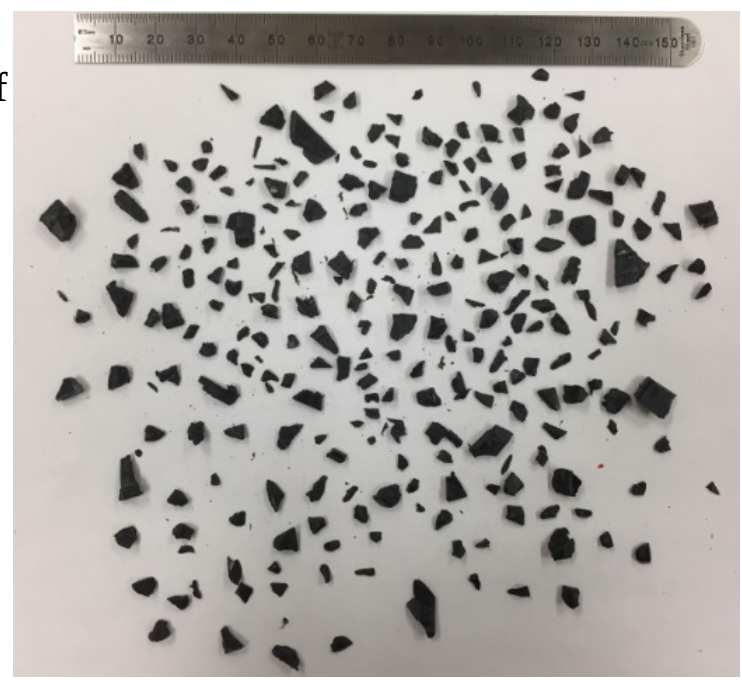

Figure 7. Particle size distribution of a handful of ABS pellets.

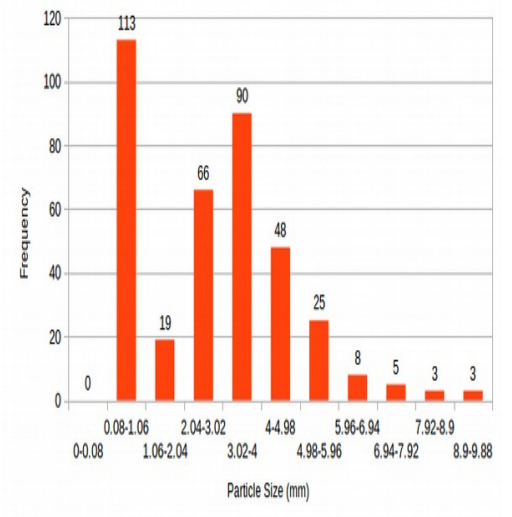


Preprint: Shan Zhong \& Joshua M. Pearce. Tightening the loop on the circular economy: Coupled distributed recycling and manufacturing with recyclebot and RepRap 3-D printing,Resources, Conservation and Recycling 128, (2018), pp. 48-58. doi: 10.1016/j.resconrec.2017.09.023

Figure 8.

Total top side areas of different particle size ranges.

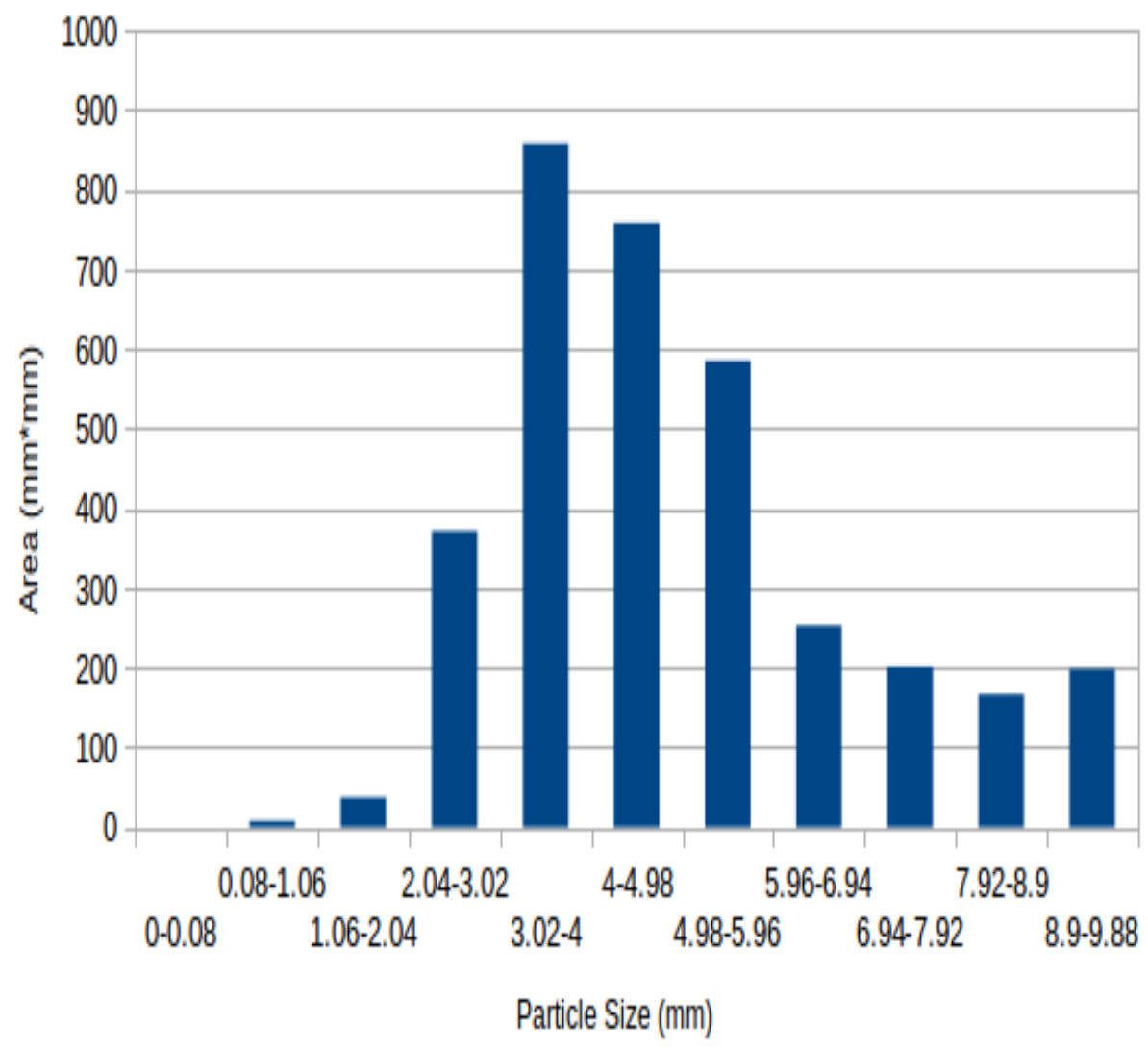

Figure 9. The filament produced by recyclebot from crushed postconsumer ABS particles. 
Preprint: Shan Zhong \& Joshua M. Pearce. Tightening the loop on the circular economy: Coupled distributed recycling and manufacturing with recyclebot and RepRap 3-D printing,Resources, Conservation and Recycling 128, (2018), pp. 48-58. doi: 10.1016/j.resconrec.2017.09.023

Figure 10. The cross sections from different parts of ABS recycled filament.

Figure 11.

The diameter distribution of recycled $\mathrm{ABS}$ filament.

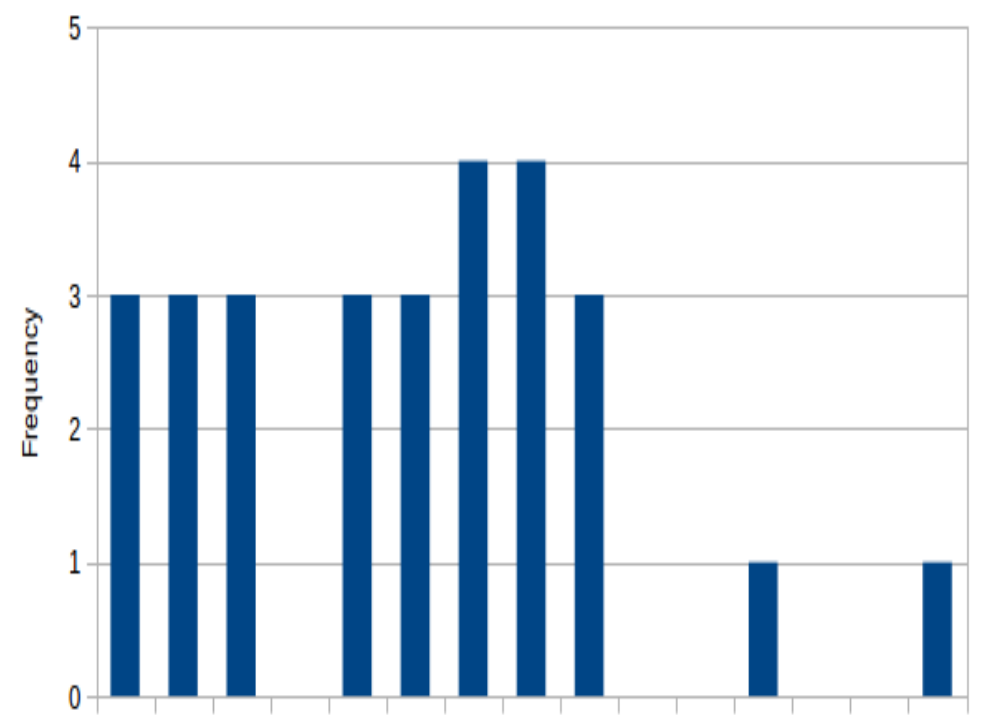

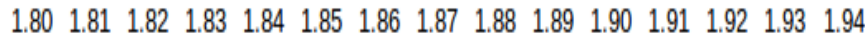
Filament Diameter (mm)

Figure 12. The camera bubble tripod printed a) components and b) assembled by the RepRap from recycled ABS filament.

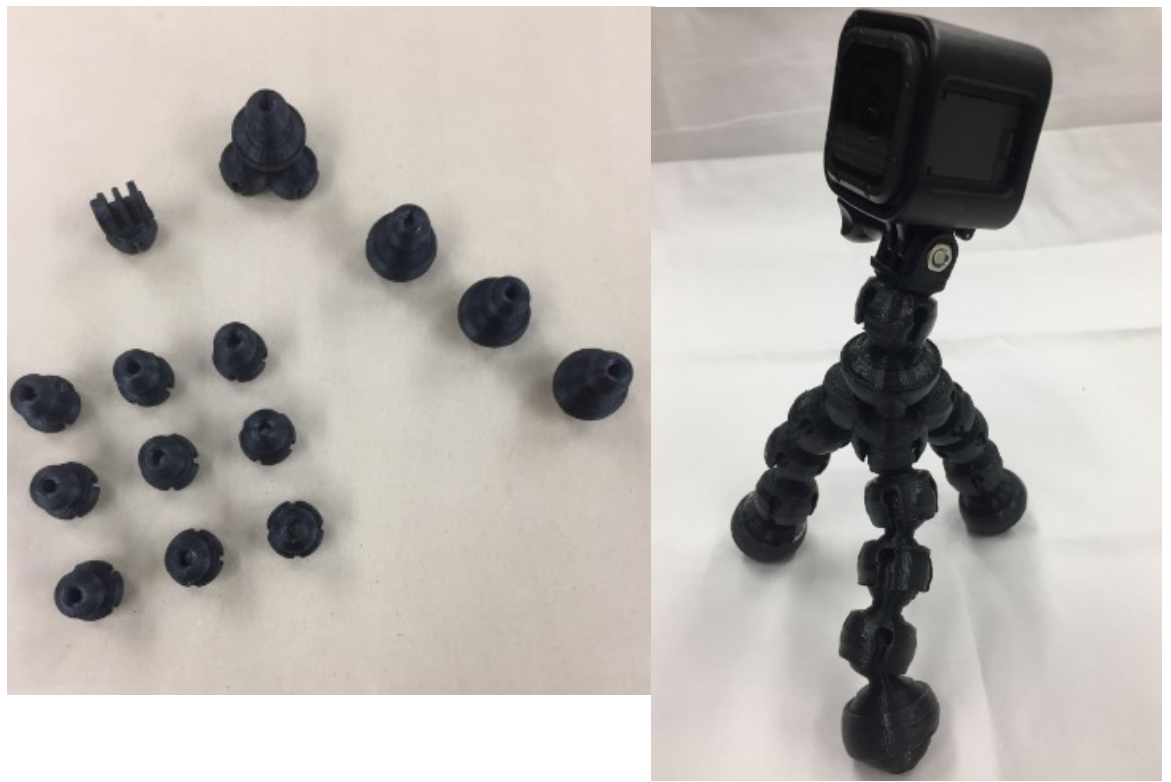


Preprint: Shan Zhong \& Joshua M. Pearce. Tightening the loop on the circular economy: Coupled distributed recycling and manufacturing with recyclebot and RepRap 3-D printing,Resources, Conservation and Recycling 128, (2018), pp. 48-58. doi: 10.1016/j.resconrec.2017.09.023

Figure 13. The SD card holder printed by the RepRap from recycled ABS filament.

Figure 14. The camera hood printed a) with camera and b) with camera by the RepRap from recycled $A B S$ filament.
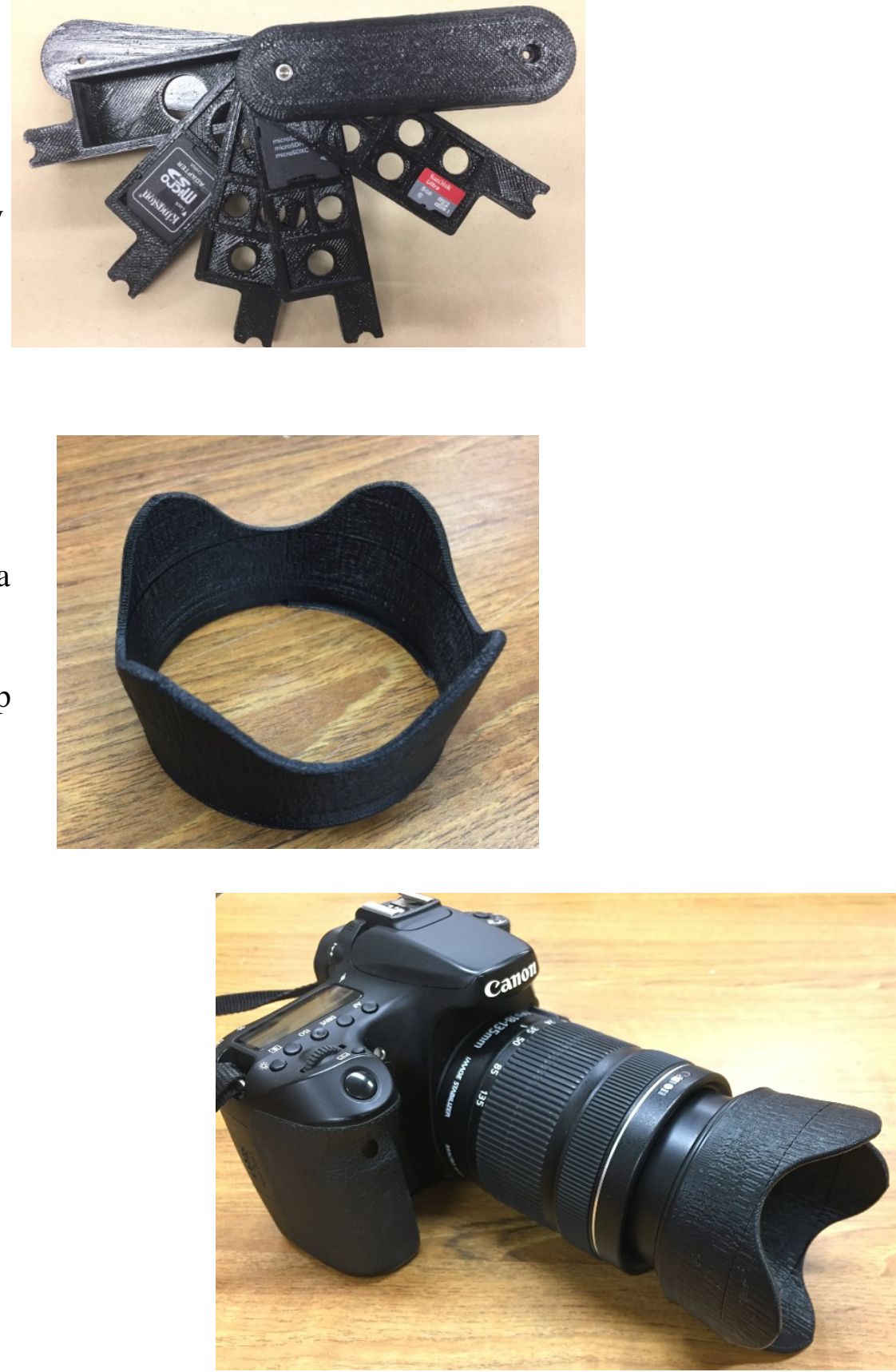
Preprint: Shan Zhong \& Joshua M. Pearce. Tightening the loop on the circular economy: Coupled distributed recycling and manufacturing with recyclebot and RepRap 3-D printing,Resources, Conservation and Recycling 128, (2018), pp. 48-58. doi: 10.1016/j.resconrec.2017.09.023 
Preprint: Shan Zhong \& Joshua M. Pearce. Tightening the loop on the circular economy: Coupled distributed recycling and manufacturing with recyclebot and RepRap 3-D printing,Resources, Conservation and Recycling 128, (2018), pp. 48-58. doi: 10.1016/j.resconrec.2017.09.023

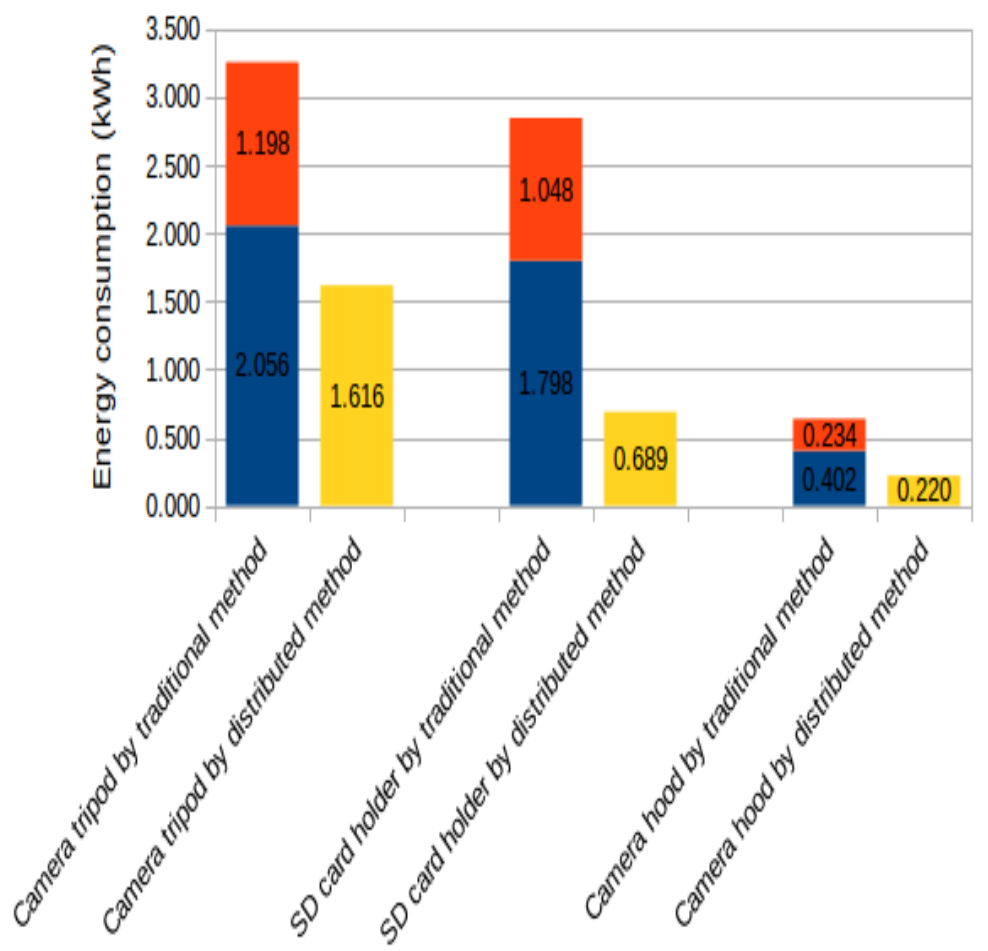

Figure 15. The energy consumption for producing a camera tripod, SD card holder and camera hood by traditional and distributed methods. 